\title{
Structure-Based Design of Benzoxazoles as new Inhibitors for D-Alanyl - D-Alanine Ligase
}

\author{
Isabelle Tytgat, ${ }^{a}$ Stéphane Vandevuer, ${ }^{b}$ Isabelle Ortmans, ${ }^{b+}$ Finton Sirockin, ${ }^{\text {c£ }}$ Evelina Colacino, ${ }^{\text {d\$ }}$ Françoise Van \\ Bambeke, ${ }^{a}$ Colette Duez, ${ }^{\mathrm{e}}$ Jacques H. Poupaert, ${ }^{\mathrm{d}}$ Paul M. Tulkens, ${ }^{\mathrm{a}}$ Annick Dejaegere, ${ }^{\mathrm{c}}$ and Martine Prévost ${ }^{\mathrm{b} *}$ \\ a Unité de Pharmacologie cellulaire et moléculaire, Université catholique de Louvain, avenue E. Mounier 73, B-1200 Brussels, \\ Belgium \\ b Structure et Fonction des Membranes Biologiques, Université Libre de Bruxelles, Boulevard du Triomphe CP 206/2, B-1050 \\ Brussels, Belgium \\ *e-mail: mprevost@ulb.ac.be; Phone: 3226502049; Fax: 3226505382 \\ Present address: UCB-Pharma, Chemin du Foriest 1, B-1420 Braine-l'Alleud, Belgium \\ c Laboratoire de Biologie et Génomique Structurales, IGBMC - UMR 7104, Ecole Supérieure de Biotechnologie de Strasbourg \\ Boulevard S. Brant, F-67400 Illkirch, France. \\ ${ }^{£}$ Present address: Novartis Institute for Biomedical Research, Postfach 4002 Basel, Switzerland \\ d Unité de Chimie pharmaceutique et radiopharmacie, avenue E. Mounier 73, Université catholique de Louvain, B-1200 Brussels, \\ Belgium. \\ \$Present address: Institut des Biomolécules Max Mousseron (IBMM) UMR 5247 CNRS - UMI \& UMII, Université de \\ Montpellier II, Place E. Bataillon, Bât 17, $2^{\text {ème }}$ étage, Montpellier Cedex, France \\ e Centre d'Ingénierie des Protéines, Institut de Chimie, B6a, Université de Liège, B-4000 Sart Tilman, Belgium.
}

Keywords: Drug design, Antibiotics, Docking, Binding free energy, D-Ala-D-Ala ligase

Received: May 11, 2009; Accepted: September 16, 2009

DOI: 10.1002/qsar.200910054

\begin{abstract}
D-Alanyl - D-alanine ligase is an enzyme which catalyzes the dimerization of D-alanine, and, as such, has an essential role in bacterial cell wall biosynthesis. It has been shown that inhibition of D-alanyl - D-alanine ligase prevents bacterial growth. D-Alanyl D-alanine ligase represents therefore a viable antimicrobial target. The 3D structure of this enzyme complexed with a phosphinophosphate inhibitor has been reported, which allows for structure-based design studies. Four softwares (LUDI, MCSS, Autodock, and Glide) developed either for fragment or full-molecule docking were compared and scored for their ability to position in the active site four prototypic ligands: two inhibitors, i.e. a phosphinophosphate derivative and D-cycloserine, D-alanine and D-alanyl - D-alanine. Best performances were obtained with Glide and MCSS. A short series of novel derivatives based on a 2-phenylbenzoxazole scaffold was designed de novo on the basis of computational data. The best compound was found to fully inhibit the D-alanyl D-alanine ligase of E. faecalis with an $I C_{50}$ of $400 \mu \mathrm{M}$.
\end{abstract}

\section{Introduction}

Emergence of bacterial isolates resistant to most current antibiotics has created an urgent need for the discovery of new agents directed against unexploited bacterial targets [1]. Among the enzymes involved in peptidoglycan synthesis, D-alanyl - D-alanine (D-Ala-D-Ala) ligase (EC 6.3.2.4), which catalyzes the dimerization of D-alanine (D-Ala) before its incorporation in late peptidoglycan precursors [2, 3 ], emerges as an interesting target, since bacteria with a mutated, inactive protein are unable to multiply unless they use an alternative pathway for cell wall synthesis [4, 5]. The reaction catalyzed by the D-Ala-D-Ala ligase involves the formation of $\mathrm{D}$-alanylphosphate followed by the nucleophilic attack of this D-alanylphosphate by the de- protonated nucleophilic amine of a second D-Ala to generate D-alanyl - D-alanine (D-Ala-D-Ala) with the release of a phosphate group $[3,6,7]$. The enzyme is strongly inhibited by phosphinophosphate [8], which mimics the tetrahedral intermediate of the reaction, and weakly by $\mathrm{D}$-cycloserine [9].

Knowledge of the protein at the molecular level could assist the rational design of novel agents as also suggested for many other potential microbial targets (see [10,11] for recent examples). The structure of E. coli D-Ala-D-Ala ligase complexed with a phosphinophosphate inhibitor has

Supporting information for this article is available on the WWW under www.qcs.wiley-vch.de 
been resolved to $2.3 \AA$ by X-ray crystallography [7]. The active site contains two distinct $\mathrm{D}$-Ala binding sites. The $\mathrm{N}$-terminal site is a high-affinity site with strict substrate specificity whereas the C-terminal site is a lower affinity site showing lower substrate specificity [9].

The development of synthetic inhibitors has recently regained interest, and new ligands have been identified [12]. Most of them were discovered by screening of chemical banks [13-16] with one active compound (3-chloro-2,2-dimethyl- $N$-[4(trifluoromethyl) phenyl]propanamide) subsequently shown by X-ray crystallography to bind in a pocket adjacent to the substrate binding site [13]. Of interest, structural knowledge of the enzyme binding pocket was helpful in most of the cases to guide the screening. Thus, inhibitors of kinases were suggested as potential inhibitors of ligases, based on structural alignments of the ATP binding site of D-alanyl - D-alanine ligase [15]. A cyclopropylbased amino acid inhibitor (2-(2-amino-2-carboxy-ethyl)1-phenyl-cyclopropanecarboxylic acid) was designed by de novo structure-based molecular modeling [17]. Recently also, virtual screening of about 2000 compounds in the Escherichia coli enzyme using the computational tool AutoDock allowed to identify 3 potent inhibitors, two of which also show a modest antibacterial activity [16].

However the potency of all ligands discovered thus far remains low, and only a few of them were evaluated for their antibacterial potency against living bacteria, making difficult to apprehend their potential interest as leads for the search of new antibacterial agents [12]. Identification of new scaffolds that could form the base of a new series of inhibitors is desirable. Moreover, a throughout assessment of the performance of the commonly used docking method on this target would be valuable for identifying new compounds, as well as improving existing ones.

In this work, we have compared 4 drug-design programs, namely LUDI [18], MCSS [19], Autodock [20] and Glide [www.schrodinger.com] [21], which have been developed and/or dedicated for either fragment-based design or fullmolecule docking. We have evaluated their capacity (a) to retrieve the positioning of the phosphinophosphate inhibitor, which was crystallized with the enzyme, and (b) to position the D-Ala substrate, the D-cycloserine inhibitor, and the reaction product D-Ala-D-Ala, which is also susceptible to act as an inhibitor [9]. The results were then used to assist in the design of new ligands for D-Ala-D-Ala ligase as well as to improve our understanding of the characteristics of the ligand-binding site interactions in this particular charged and polar active site. Along this line, as a proof of concept, a novel inhibitor was de novo designed, synthesized and evaluated for its capacity to inhibit the target enzyme and to impair the bacterial growth of reference bacterial strains.

\section{Materials and Methods}

\subsection{Complex Preparation}

The X-ray structure of D-Ala-D-Ala ligase complexed to phosphinophosphate, ADP and two $\mathrm{Mg}^{2+}$ ions (PDB code: 2DLN, [7]) was used as the target structure to endeavor the docking studies. The X-ray water and phosphinophosphate molecules were removed from the active site.

Concerning the ADP/ATP molecule also present in the active site, a difference was introduced between the docking of phosphinophosphate (see Fig. 1) and the docking of the other ligands, D-cycloserine, D-Ala substrate and the DAla-D-Ala reaction product. Indeed the phosphinophosphate inhibitor which is a phosphinic acid derivative is phosphorylated by ATP in the active site and is thus bound in the presence of two $\mathrm{Mg}^{2+}$ ions and ADP. For phosphinophosphate, ADP and the two $\mathrm{Mg}^{2+}$ ions were therefore kept in the active site pocket. However, for the D-cycloserine inhibitor, the D-ala substrate and the D-Ala-D-Ala product, ATP and not ADP should be present in the active site. To build an ATP molecule in the active site, the Protein Data Bank was screened to find all the entries involving at least one ATP molecule. Fourteen ATP structures were superimposed on the crystal ADP molecule in the binding site. The ATP molecule with a root-mean-square deviation $(R M S D)$ lower than $0.5 \AA$. and featuring the smallest number of overlaps (4 pairs) with protein atoms was kept. Minimization performed with the Protein Preparation module in the Schrodinger software (www.schrodinger.com) produces very small atomic displacements as indicated by a root-mean-square deviation of $0.2 \AA$.

\subsection{Ligand Preparation}

The ligand input files were prepared according to the following procedure. The initial structure of the three ligands, D-Ala, D-cycloserine, and D-Ala-D-Ala, was generated using Corina [22]. Two initial 3D structures of phosphinophosphate were used: one was extracted from the PDB coordinate file of the protein complex and the other generated with Corina.

For Autodock, partial charges were generated using MOPAC and the MNDO Hamiltonian. For MCSS, the partial charges were adapted from the CHARMM19 force-field [23]. For Glide the ligand partial charges were ascribed using the OPLS force-field.

\subsection{Docking and Scoring}

\subsubsection{LUDI}

LUDI is a fragment-based docking program, which was originally conceived in a de novo perspective where novel molecules are assembled from molecular fragments (Accelrys Inc, [18]). LUDI docking algorithm is completely 

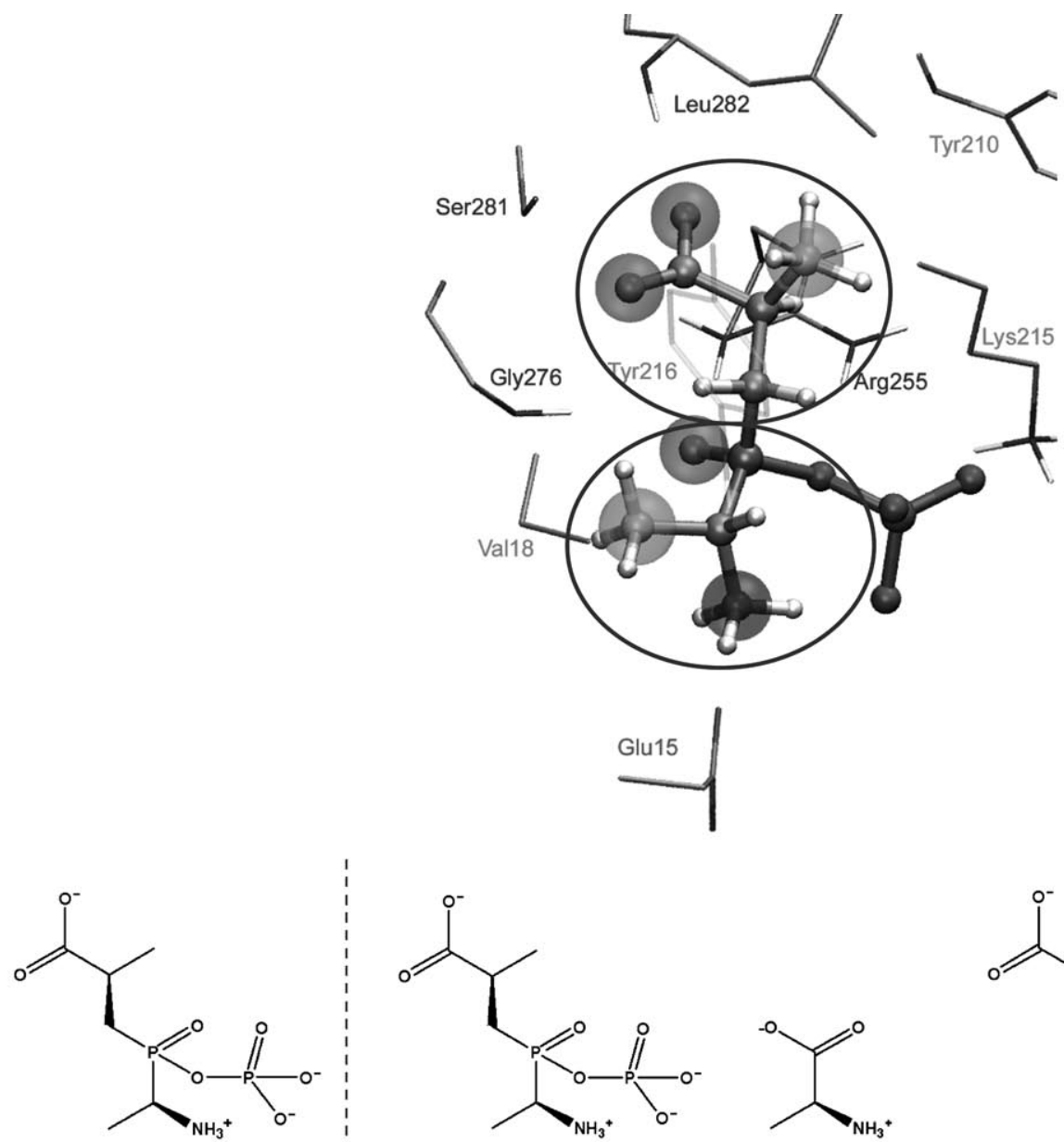<smiles>C[C@H](N)CP(=O)(OP(=O)([O-])[O-])[C@@H](C)C(=O)[O-]</smiles><smiles>C[C@H](N)C(=O)O</smiles><smiles>C[C@H](N)C(=O)N[C@@H](C)C(=O)[O-]</smiles><smiles>N[C@@H]1CONC1=O</smiles>
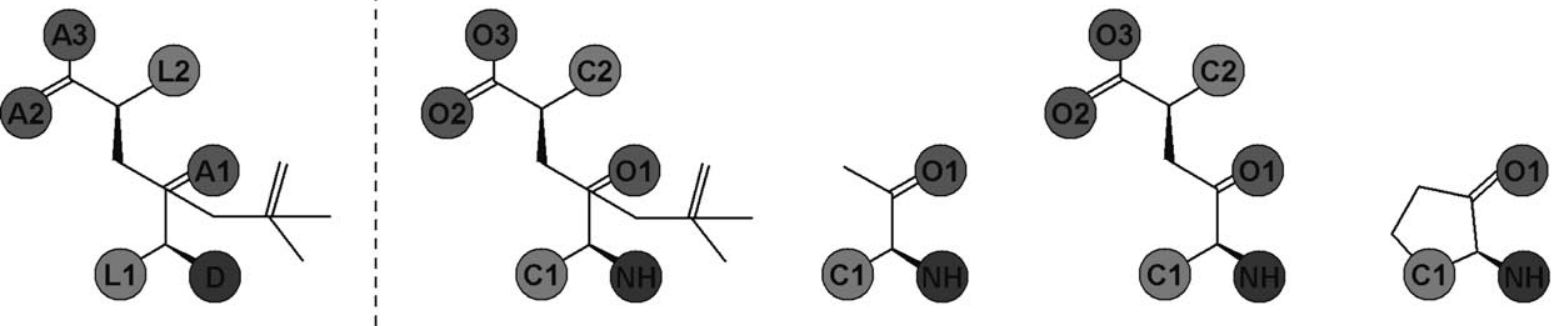

Figure 1. Top: X-ray position of the phosphinophosphate inhibitor in the active site of the D-Ala-D-Ala ligase. Phosphinophosphate is depicted as ball-and-sticks and interacting residues are represented as thick lines. Oxygens, nitrogens, carbons, phosphorus and hydrogens are colored in red, blue, cyan, ochre and white respectively. The two circles encompass the regions corresponding to the lowaffinity (top circle) and high-affinity sites (bottom circle) of the two D-Ala substrates. Bottom: Representation of the chemical structure of four ligands of the D-Ala-D-Ala ligase binding site. Left: the phosphinophosphate inhibitor with the functional sites or pharmacophoric points: A for hydrogen bond acceptor, D for hydrogen bond donor and L for lipophilic sites. Right: the four ligands, phosphinophosphate, the D-Ala substrate, the D-Ala-D-Ala reaction product and the D-cycloserine inhibitor with a schematic description of their equivalent atoms in phosphinophosphate (see text and Table 1).

based on geometric operations. Interaction sites which are discrete vectors and positions in space suitable respectively for forming hydrogen bonds or for filling a hydrophobic pocket are first determined and molecular fragments are subsequently fitted onto those interaction sites. A maxi- mum of 6 different ligand atoms prone to match interaction sites is considered. In this work the binding site was defined as a sphere with a radius of $12 \AA$ centered on the position of the central $\mathrm{CH}_{2}$ carbon of the crystal phosphinophosphate molecule. The maximum number of pose 
searches for each fragment was fixed at a value of 5000 . The docked fragments were then evaluated with the LUDI empirical scoring function [24].

\subsubsection{Autodock}

Autodock is an example of a program making use of an algorithm based on the optimization of an objective function [20]. Autodock combines a positional, orientational and conformational search engine with a grid-based method of energy evaluation. We used the genetic algorithm in Autodock to perform the global search, completed with a local search, as it has been shown that it gives efficient sampling.

Twenty independent runs were performed starting from randomly generated initial conformations of the ligand. The binding zone was defined as a parallelepiped whose center corresponds to the central $\mathrm{CH}_{2}$ carbon of the crystal phosphinophosphate molecule. Its longer side was $40 \AA$. The grid spacing was set to $0.375 \AA$. The population size was 50 and the maximum number of generations was 27000. The rate of crossover was $80 \%$ and the maximum number of individuals that automatically survive was 1 . The mutation rate was fixed to $2 \%$.

The docked poses were evaluated with the Autodock force-field scoring function.

\subsubsection{MCSS}

MCSS identifies high affinity binding sites of small fragments on a molecular surface, and can be used for de novo design, fragment screening or lead optimization [19]. It positions several hundreds copies of one single fragment in the region of the receptor to be searched. In this study, for each ligand, 500 replicas were randomly distributed in a sphere of $12 \AA$ centered on the position of $\mathrm{CH}_{2}$ atom of the phosphinophosphate molecule in the crystal structure. The initial positioning considers a minimal distance of $1.2 \AA$ between the atoms of the ligand and of the protein to avoid steric clashes in the procedure. The copies were subjected to an energy minimization combining algorithms of steepest descent and conjugated gradients using 800 steps of steepest descent followed by 20 cycles of conjugated gradients in the force-field of the protein. Interactions between the copies are omitted. A hybrid force-field, CHARMM22 for the receptor [25] and CHARMM19 for the ligands [23] was used and a distance-dependent dielectric of $4 \mathrm{r}$ was also employed. Positions of the replicas were compared after the steepest descent minimization and after each conjugated gradient cycle to eliminate replicas with a $R M S D$ of $0.2 \AA$ or less converging to a common minimum. Replicas with interaction energy with the protein above a cutoff of $500 \mathrm{kcal} / \mathrm{mol}$ were discarded. The whole procedure was repeated 20 times with a new ensemble of 500 replicas and minima converging to positions already obtained in previous cycles were eliminated.
The scoring of the MCSS minima was performed as described [26, 27]. This is a MM-PBSA based scoring [28] that sums contributions from the change in internal energy of the fragment on binding, the van der Waals interaction between the ligand and the protein, a nonpolar solvation term proportional to the loss in solvent-accessible surface area of both the protein and the ligand, and an electrostatic contribution to binding which includes the electrostatic interaction between the ligand and the protein, and the desolvation cost for each molecular partner.

\subsubsection{Glide}

The Glide (grid-based ligand docking with energetics, [www.schrodinger.com, [21]] algorithm approximates a systematic search of positions, orientations, and conformations of the ligand in the receptor binding site using a series of hierarchical filters. The shape and properties of the receptor are represented on a grid by several different sets of force-fields that provide progressively more accurate scoring of the ligand pose. The fields are computed prior to docking.

In the present work, the protein structure was prepared using the Protein Preparation Wizard in the Schrödinger software graphical user interface Maestro. The binding region was defined by a $25 \AA \times 25 \AA \times 25 \AA$ enclosed box centered on the central position of the $\mathrm{CH}_{2}$ carbon of the crystal phosphinophosphate molecule. Glide uses also a bounding box in which the ligand center must lie. This box length was set up to $12 \AA$. We used the default settings of Glide version 4.5 for the remaining parameters.

Glide XP docking protocol and scoring function were used to dock and score the poses of the different ligands.

\subsection{Evaluation of Docking Results}

Docking algorithms are generally evaluated by calculating the RMSD between the predicted pose of a given ligandtarget complex and the experimental structure of the same complex, as determined by X-ray crystallography or NMR. In our case, we had only the high resolution structure of D-Ala-D-Ala ligase with the inhibitor phosphinophosphate. There are however, enough chemical and structural similarities between the four ligands to conjecture their pose in the active site of the enzyme. To uphold this one should recall that the X-ray structure of phosphinophosphate in complex with the enzyme was used to establish the catalytic mechanism of the enzyme corroborating its similarities with the substrate D-Ala and the reaction product D-Ala-D-Ala [7]. For the small ligand D-cycloserine, no experimental structure is available either. However, despite a few differences in their donor/acceptor capabilities (see Fig. 1) D-cycloserine is generally considered as a strong structural analogue of D-Ala. Indeed in addition to its inhibitory role towards D-Ala-D-Ala ligase D-cycloserine also inhibits in a competitive manner D-alanine 
Table 1. Atoms used to measure the RMSD between the ligand and their equivalent atoms in the phosphinophosphate inhibitor (see Section 2 for details).

\begin{tabular}{lll}
\hline Ligand & Ligand atoms & Equivalent atoms \\
\hline D-Ala & $\mathrm{NH}, \mathrm{C} 1, \mathrm{O} 1$ & $\mathrm{NH}, \mathrm{C} 1, \mathrm{O} 1$ \\
D-Ala-D-Ala & $\mathrm{NH}, \mathrm{C} 1, \mathrm{O} 1, \mathrm{C} 2, \mathrm{O} 2$ & $\mathrm{NH}, \mathrm{C} 1, \mathrm{O} 1, \mathrm{C} 2, \mathrm{O} 2$ \\
Phosphinophosphate & $\mathrm{NH}, \mathrm{C} 1, \mathrm{O} 1, \mathrm{C} 2, \mathrm{O} 2$ & $\mathrm{NH}, \mathrm{C} 1, \mathrm{O} 1, \mathrm{C} 2, \mathrm{O} 2$ \\
D-Cycloserine & $\mathrm{NH}, \mathrm{C} 1, \mathrm{O} 1$ & $\mathrm{NH}, \mathrm{C} 1, \mathrm{O} 1$ \\
\hline
\end{tabular}

racemase which catalyzes the conversion of L-alanine into D-alanine [9]. Moreover, in fragment-based drug design approaches, the underlying assumption is that interesting fragments will interact with the target in a similar way either as small ligands or as part of a larger, higher affinity molecule. We therefore evaluated the performance of the fragment docking algorithms LUDI and MCSS by their ability to place key hydrogen bond donor/acceptor atoms or lipophilic groups of the small ligands D-Ala and D-cycloserine superposed to the equivalent atoms in the larger ligand phosphinophosphate. For sake of consistency, the same procedure was adopted for the larger ligand D-AlaD-Ala. The list of atoms of the ligand used for judging correct placing, together with their equivalent atoms in phosphinophosphate (template atoms) is given in Table 1.

\subsection{Bacterial Strains}

The Enterococcus faecalis JH2-2 genomic DNA was extracted to amplify the $d d l$ gene. Escherichia coli XL1-Blue or DH5 $\alpha$ was used for cloning the amplified fragment and E. coli LMG194 served to produce the recombinant enzyme.

Escherichia coli ATCC 25922, Staphylococcus aureus ATCC 25923, and Enterococcus faecalis 29212 were used for microbiological evaluation.

\subsection{Purification D-Ala - D-Ala Ligase}

\subsubsection{Construction of expression vector for Ddl ligase of Enterococcus faecalis:}

Enterococcus faecalis JH2-2 total DNA [30] was extracted as described [31]. The $d d l$ gene was amplified by PCR using the pfx polymerase (Invitrogen, Carlsbad, CA) and a pair of primers including a NcoI (5'-CGGGATCCATGGCTAAGATTATTTTGTTGTATGGCGGCAGAAG-3') and an EcoRI (5'-CGAATTCTGCAGTTTAAAACGATTCAAAGCTAAC-3') restriction sites (underlined).

The sequence of the PCR fragment was completely checked on both strains after cloning into the pGEM-T Easy plasmid (Promega). The $d d l$ gene was then subcloned into the expression $\mathrm{pBAD} / M y c$-His-A vector (Invitrogen) under the control of the $\mathrm{L}$-arabinose inducible promoter
[32] between the NcoI and EcoRI restriction sites. The resulting plasmid encoding a protein with a C-terminal 6-His tag served to transform the E. coli LMG194 expression strain (Invitrogen, [33]).

\subsubsection{Overproduction and Purification of the Ddl-His Enzyme}

Transformed bacteria were inoculated in minimal medium (RM media, Invitrogen, [34]) containing $0.2 \%$ glucose and $100 \mu \mathrm{g} / \mathrm{mL}$ ampicillin and grown at $37^{\circ} \mathrm{C}$ until an $\mathrm{OD}_{600}$ of 0.4 . At this point, $2 \% \mathrm{~L}$-arabinose was added to the culture for overnight growth at $25^{\circ} \mathrm{C}$ under shaking. All subsequent steps were performed at $4^{\circ}$. Bacteria were harvested by centrifugation, resuspended in a 20 -fold smaller volume of buffer A $\left(50 \mathrm{mM}\right.$ Hepes $\mathrm{pH} 8.0,5 \mathrm{mM} \mathrm{MgCl}_{2}, 10 \mathrm{mM}$ imidazole, $10 \%$ glycerol), and lysed by 2 successive passages in a French Press operating at 1000 psi (SLM Aminoco). Cellular debris were removed by centrifugation $(30 \mathrm{~min}$, $18000 \mathrm{~g}$ ). Ten $\mathrm{mL}$ of supernatant were incubated during 90 min with $5 \mathrm{~mL}$ of HisLink resin (Promega) under gentle agitation. Resin was pelleted by sedimentation, placed in a column and washed with $30 \mathrm{~mL}$ of buffer A. Ten $\mathrm{mL}$ of His-tagged protein were eluted by $10 \mathrm{~mL}$ of buffer B (50 mM Hepes pH 8.0, $5 \mathrm{mM} \mathrm{MgCl}_{2}, 300 \mathrm{mM} \mathrm{NaCl}$, $500 \mathrm{mM}$ imidazole, $10 \%$ glycerol). Elutate was dialyzed twice (for $2 \mathrm{~h}$ and overnight) against a 13.3-fold higher volume of buffer $\mathrm{C}(50 \mathrm{mM}$ Hepes pH 7.2, $150 \mathrm{mM} \mathrm{KCl}$, $5 \mathrm{mM} \mathrm{MgCl}_{2}, 5 \mathrm{mM} \mathrm{GSH}$ and $20 \%$ glycerol). The His-tagged protein purity was checked by SDS-polyacrylamide gel electrophoresis (Novex Tris-Glycine Gels 14\%, Invitrogen; [35]): a single band at ca. $42 \mathrm{kDa}$ was observed after Coomassie Blue staining. The purified protein concentration was measured by the method of Bradford using bovine serum albumin as standard (Quick Start Bradford Protein Assay, BioRad) and determined to be $2.24 \mathrm{mg} /$ $\mathrm{mL}$, with $48 \mathrm{mg}$ obtained from $1 \mathrm{~L}$ of induced culture. Pure fractions were stored in buffer $\mathrm{C}$ at $-80^{\circ} \mathrm{C}$.

\subsection{Enzymatic Assay and Inhibition Studies}

The activity of the His-tagged Ddl ligase (Ddl-His ${ }_{6}$ ) was determined by measuring the quantity of D-Ala-D-Ala produced from D-Ala using $\mathrm{D}-\left[{ }^{14} \mathrm{C}\right]$ Ala. Assay mixtures consisted of an assay buffer (20 mM Tris- $\mathrm{HCl} \mathrm{pH} 7.4,10 \mathrm{mM}$ $\mathrm{MgCl}_{2}, 10 \mathrm{mM} \mathrm{KCl}, 5 \mathrm{mM}$ ATP, ca. $0.03 \mu \mathrm{g} / \mu \mathrm{L}$ enzyme and $10 \mathrm{mM} \mathrm{D}-\left[1-{ }^{14} \mathrm{C}\right]$ Ala; Moravek Biochemicals, Brea, USA; diluted by cold D-Ala to a final specific activity of $0.0016 \mu \mathrm{Ci}$ /assay). Benzoxazoles were dissolved in $100 \%$ DMSO and D-cycloserine (Sigma-Aldrich, St Louis, MO, USA), used as a positive control, was dissolved in the assay buffer. They were preincubated for $5 \mathrm{~min}$ with the enzyme in the assay mixture before addition of ATP and D$\left[{ }^{14} \mathrm{C}\right]$ Ala (in all cases, the final concentration of DMSO was adjusted to $10 \% ; 0.005 \%$ Triton X-114 was added in selected cases as a means to ensure that aggregation, be- 
yond what could be seen by naked eye, could not influence the results [16]). After $30 \mathrm{~min}$ incubation at $30^{\circ}$, the reaction was stopped by $5 \mathrm{~min}$ boiling and $10 \mathrm{~min}$ centrifugation at $14500 \mathrm{~g}$ (Eppendorf Centrifuge). Two $\mu \mathrm{L}$ of the supernatant were spotted onto a $0.1-\mathrm{mm}$ microcrystalline cellulose thin-layer chromatography plate (Polygram Cel 400; Macherey-Nagel, Düren, Germany). The compounds were separated in butanol-glacial acetic acid-water $(12: 3: 5 \mathrm{vol} / \mathrm{vol} / \mathrm{vol})$ and dried at room temperature. Spots were identified by comparison with standards separated in the same conditions and revealed by staining plates with $0.25 \%$ ninhydrin in acetone and drying at $120^{\circ} \mathrm{C}$ for $3 \mathrm{~min}$. Bands associated to substrates and products were cut and the radioactivity was counted by liquid scintillation.

\subsection{MICs Determination}

MICs were determined by microdilution method in cationadjusted Muller-Hinton broth (Becton-Dickinson, NJ, USA), following the recommendations of the US Clinical and Laboratory Standards Institute (CLSI), with a final concentration of 5\% DMSO (this concentration was proven not to impair bacterial growth).

\subsection{Chemistry}

Compounds IT8, IT15, IT16 and IT21 were synthesized by standard chemistry, as reported by us [36]. IT7 and IT12 [37], and IT10 and IT11 [38] were synthesized according to published methods. The methodology used for the characterization of these compounds is described in the supporting information.

\section{Results}

\subsection{General Performance of Docking}

Each of the 4 prototypic ligands (i.e. substrate D-Ala and product D-Ala-D-Ala of the reaction, and the best two known inhibitors D-cycloserine and phosphinophosphate) was docked into the enzyme active site using the four different programs. Their performance, in terms of docking accuracy, has been evaluated by comparing the top ranked pose, which is defined as the docking solution that is ranked first in terms of energy, to their equivalent portion in the phosphinophosphate X-ray structure (see Materials and Methods Section, Table 1 and Fig. 1). Prediction accuracy was measured by the Root Mean Square Deviation (RMSD) between the coordinates of ligand atoms and those of the matching pharmacophore points (Table 2). Usually a prediction is considered as successful if the $R M S D$ value is lower than or equal to $2 \AA$ [39]. For low molecular weight ligands, i.e. D-Ala and D-cycloserine, MCSS outperformed the other docking programs. For larger ligands, D-Ala-D-Ala and phosphinophosphate, Glide was identified as the most accurate program. Both Glide and MCSS produced RMSD values lower than $2 \AA$ for three out of the four ligands. For the small ligands DAla and D-cycloserine, the prediction with LUDI was found correct for D-Ala but less accurate for D-cycloserine (see Table 2).

Docking performance was also assessed by considering the top 10-ranked poses and selecting out those with the pose having the lowest $R M S D$ as compared to its equivalent portion in the phosphinophosphate X-ray structure (Table 3). Both Glide and MCSS found a best pose with a RMSD inferior or equal to $1 \AA$ for all ligands. Autodock performs also quite well, with all best poses at an RMSD smaller than $2 \AA$. LUDI generates only one pose; therefore the top (Table 2) and best (Table 3) poses are equivalent.

Numerical measures such as $R M S D$ need careful interpretation and make difficult to draw conclusions of general applicability [40]. The RMSD measures were then supplemented here by interaction-based measures and visual inspection of the docking poses. The interactions formed between the ligands and the protein atoms were analyzed to help interpreting the $R M S D$ values of the best poses. Table 1 in supplementary material lists the interactions formed between the ligands and the amino acid residues in the binding pocket. The latter was divided in two different regions corresponding to the high- and low D-Ala affinity sites (see Fig. 1). The interactions made by the pharmacophoric sites of the phosphinophosphate shared with each ligand in the crystal structure are also listed for sake of comparison. These results confirm the conclusions based on the RMSD values, namely that Glide and MCSS are successful in reproducing a ligand pose forming a fairly large number of expected interactions with the target

Table 2. RMSD measures between the top-ranked pose for the four ligands generated by the four different docking programs. Two values are given for the phosphinophosphate. The value on the left corresponds to that obtained for the initial X-ray structure and that on the right for a 3D initial structure generated by Corina (see Sec. 2). See Table 1 for the list of atoms used to compute the RMSD.

\begin{tabular}{lllll}
\hline Top pose $R M S D / \AA$ & D-Ala-D-Ala & Phosphinophosphate & D-Cycloserine \\
\hline LUDI & D-Ala & n/a & n/a & 3.14 \\
MCSS & 1.26 & 2.26 & $1.77-2.02$ & 1.31 \\
Autodock & 0.72 & 3.30 & $0.86-1.6$ & 3.26 \\
Glide & 1.61 & 0.52 & $0.92-0.70$ & 2.72 \\
\hline
\end{tabular}


Table 3. $R M S D$ measures between the best poses for the four ligands generated by the four different docking programs and the $\mathrm{X}$ ray structure of phosphinophosphate. Two values are given for the phosphinophosphate. The value on the left corresponds to that obtained for the initial X-ray structure and that on the right for a 3D initial structure generated by Corina (see Sec. 2). See Table 1 for the list of atoms used to compute the RMSD.

\begin{tabular}{lllll}
\hline Best pose RMSD $(\AA)$ & D-Ala & D-Ala-D-Ala & Phosphinophosphate & D-Cycloserine \\
\hline LUDI & 1.26 & n/a & n/a & 3.14 \\
MCSS & 0.72 & 0.93 & $0.90-2.02$ & 0.93 \\
Autodock & 1.61 & 1.41 & $0.85-1.1$ & 1.21 \\
Glide & 0.52 & 0.42 & $0.61-0.65$ & 1.00 \\
\hline
\end{tabular}

binding site. This is particularly striking when one looks at the best pose in terms of $R M S D$ and interactions. However, the exclusive analysis of results based on interactions suggests that the best poses predicted by Autodock are also in good agreement with those observed in the crystal structure of phosphinophosphate.

\subsection{Rational Design of Inhibitors}

Based on the results obtained so far, we studied the binding of small ligands to the D-Ala-D-Ala ligase binding site using MCSS method. The choice of the small ligands was guided by the different physical categories of interactions encountered in enzyme-ligand complexes and also by the type of interactions detected in our analysis heretofore. Ten different fragments were selected. They are chemical fragments of larger organic molecules with different character such as charged, polar, aromatic, hydrophobic and aliphatic groups. The propane molecule was used as a pure aliphatic group, benzene, oxazole, pyrizadine and 5-methyl imidazole as aromatic groups. Methylammonium and propylguanidium ion were chosen as positively charged groups and acetate as a negatively charged group. In addition ethanol, cyclopentanone and barbituric acid were also studied. They contain at least one polar chemical function. The pharmacophore points used in this process are shown in Figure 1.

The positions adopted by methylammonium could be subdivided in two groups, one of which is located slightly below the $\mathrm{D}$ pharmacophore point matching the $\mathrm{NH}_{3}^{+}$of the phosphinophosphate inhibitor and interacts with Glu15 and Glu68. For propylguanidium ion there is a large dispersion of the positions in the binding site. The binding energy is more favorable for geometries with the guanidium group located at the bottom of the active site so as to make salt bridges with Glu15, Glu 68 and the ATP molecule. The positions occupied by the acetate group can also be clustered in 2 groups, one of which matches the A2 and A3 pharmacophore points corresponding to the carboxylic group of the phosphinophosphate inhibitor. Barbituric acid also displays a dispersion of the positions. Those corresponding to the most favorable energy form two hydrogen bonds with Arg255 and Leu252. Cyclopentanone compound positions preferentially in a lateral pocket whose entrance is located next to the $\mathrm{CH}_{3}$ side chain of the C-ter- minal portion of phosphinophosphate (see Fig. 1). Its carbonyl group hydrogen bonds with Lys215. The positions of propane sample 3 privileged sites. The first corresponds to the L2 pharmacophoric site. The second to the lateral pocket next to the second $\mathrm{CH}_{3}$ side chain group of phosphinophosphate in the crystal structure and the third superposes to the L1 pharmacophoric site. The ethanol molecule also samples three different sites: the most energetically favorable site is found in the lateral pocket and the geometries feature a hydrogen bond with Lys215. The different positions occupied by benzene, oxazole and pyridazine aromatic nuclei cover the whole binding region of the phosphinophosphate inhibitor. In one of the positions, oxazole forms hydrogen bonds with Tyr216 and Arg255.

Our docking studies suggest that an inhibitor candidate should interact with polar groups (Glu15, Glu68, Arg255 and possibly Ser281). This could be achieved via hydrogen bonds or salt bridges, but the corresponding inhibitor would be far too polar to cross biological membranes. The charge-charge interactions could, however, be replaced by charge-dipole or charge-quadrupole interactions. The latter could be brought by an aromatic moiety, which would also allow decreasing the flexibility of the ligand (and, thereby, the corresponding entropy penalty), and the desolvation energy [41]. An aromatic moiety in the ligand could be stabilized by aromatic-aromatic interactions with Tyr210 and/or Tyr216, and could also increase the affinity towards the active site. This could help in rationally restricting the chemical space of molecules when, for instance, screening databases of ligands. To avoid reproducing too strongly charged or polar ligands, we decided to opt for aromatic fragments such as benzene and oxazole to build a scaffold molecule that allowed favorable interactions with the main residues of the binding site.

The MCSS results describe a pharmacophore mapping of the binding site that can be used for lead identification. A visual inspection of the minima with the most favorable MM-PBSA scoring (see Materials and Methods Section) from each cluster of these fragments (see Fig. 1 in supporting information) prompted us to design a benzoxazole molecule. This compound was docked into the binding site using Glide. The three most favorable positions were found to superpose to the C-terminal portion of the crystal location of phosphinophosphate and scored to a value of about $-6 \mathrm{kcal} / \mathrm{mol}$. In order to fill the remaining empty 
Table 4. Inhibitory activity of benzoxazoles towards Ddl and antibacterial activity against E. coli, S. aureus or E. faecalis.

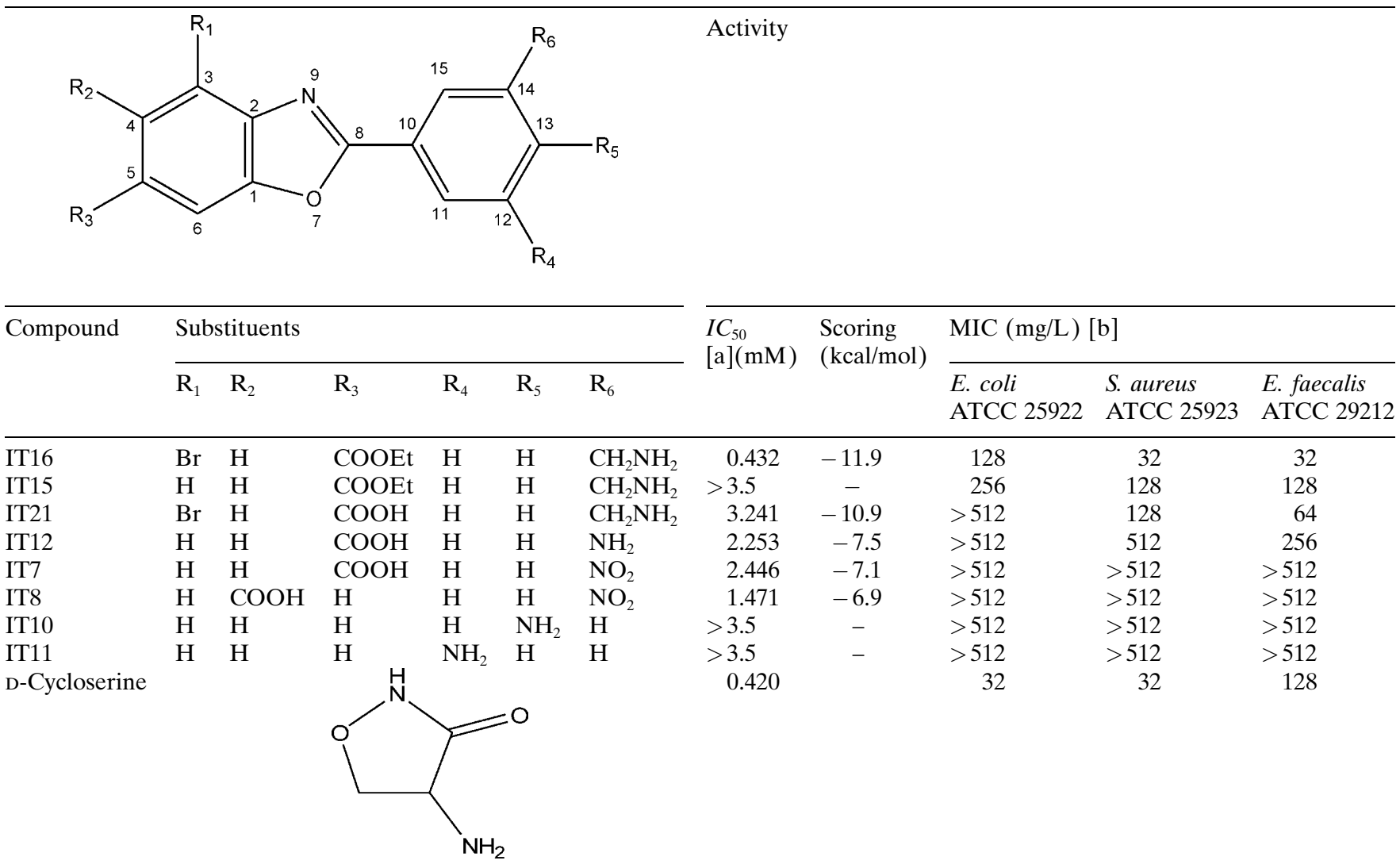

[a] determined in the presence of $10 \%$ DMSO for all molecules

[b] geometric dilutions, determined in the presence of 5\% DMSO for all molecules

part of the binding pocket, a phenyl group was added to the benzoxazole scaffold. A 2-phenyl benzoxazole molecule was then designed. Variations of substituents were proposed on the benzene ring of the benzoxazole and on the phenyl ring, some of them suggested by MCSS mapping of acetate and methylammonium fragments.

A series of 15 benzoxazoles were produced and tested first for their capacity to inhibit the purified Ddl at a fixed concentration of $0.6 \mathrm{mM}$ (i. e. approx. the $I C_{50}$ of D-cycloserine in the conditions of our assay). This first screening allowed selecting 5 active molecules, which were used for determination of their own $I C_{50}$ and of antimicrobial activity in comparison with D-cycloserine. Three less active compounds with closely related structures were also examined in these studies as controls. Table 4 shows that IT16 is the most active of the benzoxazoles tested, with an $\mathrm{IC}_{50}$ close to that of D-cycloserine, and MICs against Gram-positive cocci similar or lower than those of D-cyloserine. Among the other products, IT21, the nonesterified form of IT16, had a 8-fold higher $I C_{50}$ and also higher MICs while IT15, which lacks the Br substituent, had a detectable antimicrobial effect but a much higher $I C_{50}$. IT7, 8 and 12 were inactive against bacteria despite an inhibitory activity slightly better than that of IT21. IT10 and IT11, which lack a carboxylate function, proved ineffective on the enzyme as well as on bacteria. To rule out a possible role of aggregation of the benzoxazole molecules in the reduction of enzymatic activity observed at high concentrations, one compound (IT8) was retested in the presence of $0.005 \%$ Triton X-114 [16]. No difference was seen with the data obtained in the absence of detergent.

The five active molecules were docked back into the binding site using Glide. Their scoring values are given in Table 4. IT16 features a higher scoring value relative to all the other four molecules in agreement with the experimental data. This may arise from the larger number of salt bridges and hydrogen bonds interactions that IT16 makes with the active site residues (Fig. 2). However the observed difference in $I C_{50}$ between IT16 and IT21 is not fully picked out by the docking study though IT21 is predicted to have an affinity slightly lower than IT16. Both molecules make about the same favorable interactions. Some differences however are observed when dissecting the energetic contributions with more favorable van der Waals and lipophilic interactions for IT16 relative to IT21. 


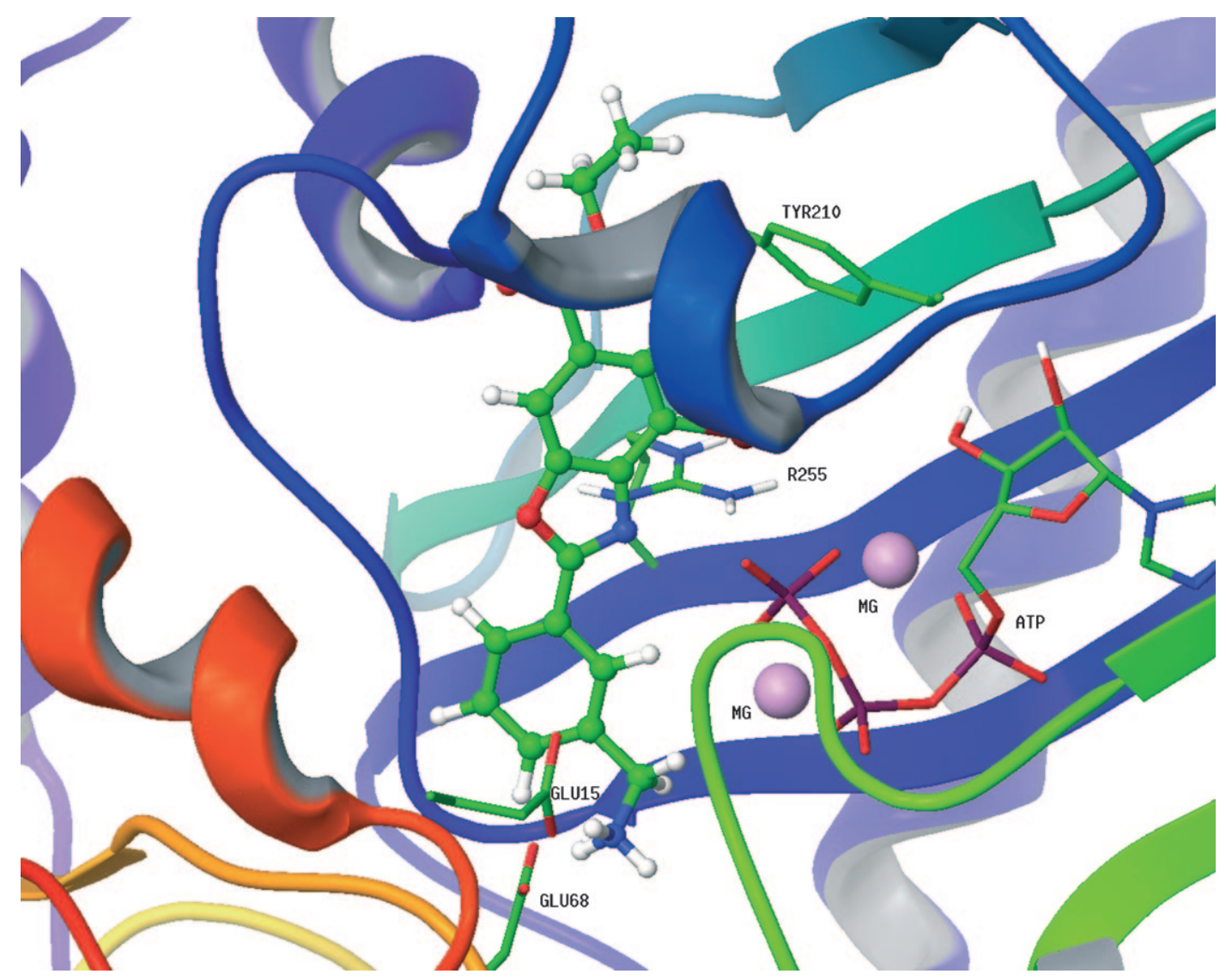

Figure 2. Docked pose of IT16 in the E. coli D-Ala-D-Ala ligase enzymatic pocket. IT16 is depicted as ball-and-sticks and interacting residues and ATP are represented as thick lines. The two magnesium ions are depicted as pink spheres. Oxygens, nitrogens, carbons, phosphorus and hydrogens are colored in red, blue, green, magenta and white respectively.

\section{Discussion}

Previous reports [42-47] have pointed out that the performances of most docking tools are very target-dependent. In the case of D-Ala-D-Ala ligase, the programs that performed best were Glide and MCSS, with Glide giving the best results for the top-ranked positioning of larger ligands and the best pose for 3 out of 4 ligands, and MCSS producing best top positionings for smaller ligands and best pose for D-cycloserine. Even though Autodock performed not as well as Glide and MCSS, it predicted poses with $R M S D$ values all below $2 \AA$ for the four ligands, and protein-ligand interactions were in fair agreement with the crystal structure of the protein-phosphinophosphate complex. In agreement with other studies [39, 47] our results also clearly show the necessity of saving several topranked poses to improve the rate of successful predictions.

The efficiency of Glide was shown in several studies [42, $44-47]$. Loss of capacity was however observed when facing hydrophilic binding sites [45]. It was thus important to verify its performance for docking the highly polar binding site of D-Ala-D-Ala ligase.

MCSS was originally devised for docking small, mostly rigid ligands, which is further confirmed by the present data. In our hands, MCSS also gave good results for the larger, more flexible ligands phosphinophosphate and DAla-D-Ala. Since MCSS does not perform extensive conformational search of the unbound ligand conformation, its good performance was most likely linked to a favorable starting conformation of these two ligands. This is shown for phosphinophosphate, for which two different structures were used. MCSS produces the best result for the structure extracted from the crystal complex structure (see Table 3). For D-Ala-D-Ala, its initial structure was generated in an extended conformation close to that expected in the complex and the MCSS good performance may have arisen by accident (see Materials and Methods section). Choosing an initial structure in a conformation close to that bound to the enzyme can be particularly helpful when using a program such as MCSS since its search algorithm is based on energy minimization. This, in contrast, should not influence so much the results produced by Autodock or Glide (see Table 3).

This study has allowed us to rationally design a new family of Ddl inhibitors. IT16, the best compound, inhibits the D-Ala-D-Ala ligase with an $I C_{50}$ of $400 \mu \mathrm{M}$. We did not find however a clear correlation between scoring by Glide and potency as ligase inhibitor for the different tested 
compounds. This was also the case in the study of Kovac et al. [14], who found $K_{i}$ values ranging from 42 to $218 \mu \mathrm{M}$ for inhibitors scored by AutoDock at -16.02 and $-18.34 \mathrm{kcal} / \mathrm{mol}$, respectively and a $K_{i}$ value of $11 \mu \mathrm{M}$ for an inhibitor scored at $-8.87 \mathrm{kcal} / \mathrm{mol}$. This further illustrates that docking programs are helpful in predicting the capacity of a molecule to fit in the enzymatic cavity of Ddl, but are less potent in ranking their binding affinity.

Of interest, the most powerful designed inhibitor IT16 in our series is also the most active on bacterial growth of Gram-positive bacteria. Correlation is not perfect, however as IT8, which is only 3 times less potent on ligase than IT16, is unable to prevent bacterial growth. Among the families of inhibitors described so far, only a few were tested for their antimicrobial activity [12]. In these studies also, there was no clear correlation between enzymatic inhibition and antimicrobial potency, as illustrated for (i) diazenecarboxamides [14], which show $I C_{50}$ towards Ddl 20 times lower than D-cycloserine but higher MICs, (ii) the natural compound quercetin [49], which shows lower $I C_{50}$ and $K_{i}$ values than apigenin towards the $H$. pylori enzyme, but 8 times higher MIC towards the bacterium, or (iii) two molecules from the NCI database [16] which display similar MIC towards $S$. aureus but $\mathrm{K}_{i}$ differing by one order of magnitude. Beyond intrinsic inhibitory potency, other parameters, such as the ability of the molecules to reach their intracellular target are also critical and probably explain these discrepancies. This will need to be explored in detail, as differences in target accessibility relate to lack of diffusibility as well as to active efflux or to intracellular degradation of the study compounds.

Although benzoxazoles were already known in the literature for an antifungal $[50,51]$ anticancer [52], antihelmintic [53], or even antibiotic [54, 55] activity, there was no previous indication of their potential target in bacteria. Our experimental data therefore underline the performance of the tools used and also open perspectives for further lead optimization.

\section{Acknowledgements}

IT and SV were granted by the Belgian Fonds pour la Recherche dans l'Industrie et l'Agriculture (FRIA). MP and FVB are Maîtres de Recherche and CD is Chercheur Qualifié of the Belgian Fonds de la Recherche Scientifique (F.R.S.-FNRS). EC was postdoctoral fellow supported by the Pôle d'Attractions Interuniversitaires (PAI) Program of the Federal Belgian government (P5/33). AD thanks the University of Strasbourg, France, the French institutes CNRS (Centre National de la Recherche Scientifique) and INSERM (Institut National de la Santé et de la Recherche Médicale) for financial support. This work was supported by the Belgian Fonds pour la Recherche Scientifique Médicale (grant no. 3.4.597.06), the Bioval program from the Région Wallonne, and the Belgian Program on Interuni- versity Poles of Attraction initiated by the Belgian state, Prime Minister's Office, Science Policy Programming (P5/ 33 and PAI6/19).

\section{References}

[1] M. T. Black, J. Hodgson, Adv. Drug Deliv. Rev. 2005, 57, $1528-1538$.

[2] F. C. Neuhaus, Biochem. Biophys. Res. Commun. 1960, 3, $401-405$.

[3] C. T. Walsh, J. Biol. Chem. 1989, 264, 2393-2396.

[4] M. Baptista, F. Depardieu, P. Reynolds, P. Courvalin, M. Arthur, Mol. Microbiol. 1997, 25, 93-105.

[5] F. Van Bambeke, M. Chauvel, P. E. Reynolds, H. S. Fraimow, P. Courvalin, Antimicrob. Agents Chemother. 1999, 43, $41-47$.

[6] H. A. Carlson, J. M. Briggs, J. A. McCammon, J. Med. Chem. 1999, 42, 109-117.

[7] C. Fan, P. C. Moews, C. T. Walsh, J. R. Knox, Science 1994, 266, 439-443.

[8] W. H. Parsons, A. A. Patchett, H. G. Bull, W. R. Schoen, D. Taub, J. Davidson, P. L. Combs, J. P. Springer, H. Gadebusch, B. Weissberger, M. E. Valliant, T. N. Mellin, R. D. Busch, J. Med. Chem. 1988, 31, 1772-1778.

[9] L. E. Zawadzke, T. D. Bugg, C. T. Walsh, Biochemistry 1991, 30, $1673-1682$.

[10] M. Moche, G. Schneider, P. Edwards, K. Dehesh, Y. Lindqvist, J. Biol. Chem. 1999, 274, 6031-6034.

[11] G. Schluckebier, P. Zhong, K. D. Stewart, T. J. Kavanaugh, C. J. Abad-Zapatero, Mol. Biol. 1999, 289, 277-291.

[12] I. Tytgat, E. C. Colacino, P. M. Tulkens, J. H. Poupaert, M. Prévost, F. Van Bambeke, Curr. Med. Chem. 2009, 16: $2566-2580$.

[13] S. Liu, J. S. Chang, J. T. Herberg, M. M. Horng, P. K. Tomich, A. H. Lin, K. R. Marotti, Proc. Natl. Acad. Sci. USA 2006, 103, 15178-15183.

[14] A. Kovac, V. Majce, R. Lenarsic, S. Bombek, J. M. Bostock, I. Chopra, S. Polanc, S. Gobec, Bioorg. Med. Chem. Lett. 2007, 17, 2047-2054.

[15] G. Triola, S. Wetzel, B. Ellinger, M. Koch, K. Hübel, D. Rauh, H. Waldmann, Bioorg. Med. Chem. 2009, 17, 10791087.

[16] A. Kovac, J. Konc, B. Vehar, J. M. Bostock, I. Chopra, D. Janezic, S. Gobec J. Med. Chem. 2008, 51, 7442-7448.

[17] G. E. Besong, J. M. Bostock, W. Stubbings, I. Chopra, D. I. Roper, A. J. Lloyd, C. W. Fishwick, A. P. Johnson, Angew. Chem. Int. Ed. Engl. 2005, 44, 6403-6406.

[18] H. J. Bohm, J. Comput. Aided Mol. Des. 1992, 6, 593-606.

[19] A. Miranker, M. Karplus, Proteins 1991, 11, 29-34.

[20] G. M. Morris, D. S. Goodsell, R. S. Halliday, R. Huey, W. E. Hart, R. K. Belew, A. J. Olson, J. Comput. Chem. 1998, 19, $1639-1662$.

[21] R. A. Friesner, J. L. Banks, R. B. Murphy, T. A. Halgren, J. J. Klicic, D. T. Mainz, M. P. Repasky, E. H. Knoll, M. Shelley, J. K. Perry, D. E. Shaw, P. Francis, P. S. Shenkin, J. Med. Chem. 2004, 47, 1739-1749.

[22] J. Sadowski, J. Gasteiger, Chem. Rev. 1993, 93, 2567-2581.

[23] B. R. Brooks, R. E. Bruccoleri, B. D. Olafson, D. J. States, S. Swaminathan, M. Karplus, J. Comput. Chem. 1983, 4, $187-$ 217.

[24] H. J. Bohm, J. Comput. Aided Mol. Des 1998, 12, 309-323. 
[25] A. D. MacKerell, D. Bashford, M. Bellott, R. L. Dunbrack, J. D. Evanseck, M. J. Field, S. Fischer, J. Gao, H. Guo, S. Ha, D. Joseph-McCarthy, L. Kuchnir, K. Kuczera, F. T. K. Lau, C. Mattos, S. Michnick, T. Ngo, D. T. Nguyen, B. Prodhom, W. E. Reiher, B. Roux, M. Schlenkrich, J. C. Smith, R. Stote, J. Straub, M. Watanabe, J. Wiorkiewicz-Kuczera, D. Yin, M. Karplus, J. Phys. Chem. B 1998, 102, 3586-3616.

[26] F. Sirockin, C. Sich, S. Improta, M. Schaefer, V. Saudek, N. Froloff, M. Karplus, A. Dejaegere, J. Am. Chem. Soc. 2002, 124, 11073-11084.

[27] M. Schechner, F. Sirockin, R. H. Stote, A. P. Dejaegere, J. Med. Chem. 2004, 47, 4373-4390.

[28] P. A. Kollman, I. Massova, C. Reyes, B. Kuhn, S. Huo, L. Chong, M. Lee, T. Lee, Y. Duan, W. Wang, O. Donini, P. Cieplak, J. Srinivasan, D. A. Case, T. E. Cheatham III, Acc. Chem. Res. 2000, 33, 889-897.

[29] M. D. Eldridge, C. W. Murray, T. R. Auton, G. V. Paolini, R. P. Mee, J. Comput. Aided Mol. Des 1997, 11, 425-445.

[30] A. E. Jacob, S. J. Hobbs, J. Bacteriol. 1974, 117, 360-372.

[31] C. Le Bouguenec, G. de Cespedes, T. Horaud, J. Bacteriol 1990, 172, 727-734.

[32] L. M. Guzman, D. Belin, M. J. Carson, J. Beckwith, J. Bacteriol. 1995, 177, 4121-4130.

[33] J. M. Fleckenstein, K. Roy, J. F. Fischer, M. Burkitt, Infect Immun. 2006, 74, $2245-2258$.

[34] G. A. Gambetta, J. C. Lagarias, Proc. Natl. Acad. Sci. USA 2001, 98, 10566-10571.

[35] U. K. Laemmli, Nature 1970, 227, 680-685.

[36] J. H. Poupaert, M. Prévost, S. Vandevuer, F. Van Bambeke, E. Colacino, I. Tytgat P. M. Tulkens, PTC Int. Appl. 2009, WO2008EP6810020081219.

[37] S. M. Courtney, P. A. Hay, K. Heleyova, D. Ilavsky, V. Bobosik, N. Pronayova, PCT Int. Appl. 2003, WO 2003074516.

[38] K. Heleyova, D. Ilavsky, V. Bobosik, N. Pronayova, Coll. Czechoslovak. Chem. Commun. 1996, 61, 371-380.
[39] R. Wang, Y. Lu, S. Wang, J. Med. Chem. 2003, 46, 22872303.

[40] J. C. Cole, C. W. Murray, J. W. Nissink, R. D. Taylor, R. Taylor, Proteins 2005, 60, 325-332.

[41] J. P. Gallivan, D. A. Dougherty, J. Am. Chem. Soc. 2000, $122,870-874$.

[42] M. D. Cummings, R. L. DesJarlais, A. C. Gibbs, V.; Mohan, E. P. Jaeger, J. Med. Chem. 2005, 48, $962-976$.

[43] E. Kellenberger, J. Rodrigo, P. Muller, D. Rognan, Proteins 2004, 57, 225-242.

[44] M. Kontoyianni, L. M. McClellan, G. S. Sokol, J. Med. Chem. 2004, 47, 558-565.

[45] E. Perola, W. P. Walters, P. S. Charifson, Proteins 2004, 56, $235-249$.

[46] R. Wang, Y. Lu, X. Fang, S. Wang, J. Chem. Inf. Comput. Sci. 2004, 44, 2114-2125.

[47] G. L. Warren, C. W. Andrews, A. M. Capelli, B. Clarke, J. LaLonde, M. H. Lambert, M. Lindvall, N. Nevins, S. F. Semus, S. Senger, G. Tedesco, I. D. Wall, J. M. Woolven, C. E. Peishoff, M. S. Head, J. Med. Chem. 2006, 49, 5912-5931.

[48] D. J. Diller, R. Li, J. Med. Chem. 2003, 46, 4638-4647.

[49] D. Wu, Y. Kong, C. Han, J. Chen, L. Hu, H. Jiang, X. Shen, Int. J. Antimicrob Agents 2008, 32, 421-426.

[50] P. R. Duchowicz, M. G. Vitale, E. A. Castro, M. Fernández, J. Caballero, Bioorg. Med. Chem. 2007, 15, 2680-2689.

[51] E. Sener, I. Yalcin, E. Sungur, QSAR 1991, 10, 223-228.

[52] M. S. Chua, D. F. Shi, S. Wrigley, T. D. Bradshaw, I. Hutchinson, P. N. Shaw; D. A. Barrett, L. A. Stanley, M. F. Stevens, J. Med. Chem. 1999, 42, 381-392.

[53] L. I. Denisova, V. M. Kosareva, I. G. Solonenko, Byul. Vses. In-ta Gel'mintol. 1979, 24, 25.

[54] I. Yalcin, E. Sener, T. Ozden, S. Ozden, A. Akin, Eur. J. Med. Chem. 1990, 25, 705-708.

[55] I. Yalcin, E. Sener, Int. J. Pharm. 1993, 98, 1-8. 